Note

\section{Specificities of Bio-antimutagens in Plant Kingdom}

\author{
Ryuichiro IsHII, Kentaro YoshIKaWA, \\ Hiroyuki Minakata, ${ }^{*}$ Hajime Komura* \\ and Tsuneo KADA** \\ Department of Food and Nutrition, Kinki University, \\ Higashi-Osaka, Osaka 577, Japan \\ *Suntory Institute for Bio-Organic Research, \\ Wakayama-dai, Mishima-gun, Osaka 618, Japan \\ **Department of Induced Mutation, National Institute \\ of Genetics, Mishima, Shizuoka 411, Japan
}

Received April 23, 1984

Desmutagens are defined as agents that inactivate chemical mutagens by working directly on them. ${ }^{1)}$ On the other hand, we like to call bio-antimutagens those agents suppressing cellular mutagenesis. Bio-antimutagens of diverse chemical types have been recently isolated from different plants and characterized, for example, protoanemonin from Ranunculus, ${ }^{2)}$ cinnamaldehyde from cinnamon, ${ }^{3)}$ and enmein from Enmeiso. ${ }^{4)}$ Since these antimutagens were isolated with the criterion of reducing the frequency of $\mathrm{UV}$-induced mutations of $E$. coli $\mathrm{B} / \mathrm{r}$ trp involving the SOS DNA-repair function, it is interesting to know if antimutagenic factors of similar specificities exist universally in the plant kingdom. The other implication of surveying them in plants relates to their functions. We previously found that a typical bio-antimutagen such as cobaltous chloride working in UV-induced mutations in the above $E$. coli $\mathrm{B} / \mathrm{r}$ strain also antagonized to a high frequency mutations due to a temperature-sensitive DNApolymerase III in B. subtilis. ${ }^{5,6)}$ Therefore, it is interesting to examine if the UV-induced SOS repair might involve a kind of DNA-replicating polymerase, as suggested by Bridges et al. ${ }^{7 \text { ) }}$

We checked the bio-antimutagenic capacities of extracts obtained from 303 different plants (Table I) with criteria of suppressing UV-induced mutations in E. coli $\mathrm{B} / \mathrm{r}$ WP2 (trp) and the mutator activity in B. subtilis NIG1125 (his $m e t)$. The spot tests were carried out using both strains for the experimental procedures which have already been described by Kakinuma et al. ${ }^{3,4)}$ For preparation of crude extracts, $3.5 \mathrm{~g}$ of plant tissues (mainly leaves and stems) of each plant were immersed in $14 \mathrm{ml}$ of $80 \%$ methanol with vigorous stirring. part of the extract (usually $50 \mu \mathrm{l}$ ) was soaked into a paper disk and placed on the surface of an MB agar plate on which test bacterial cells were spread. Appearance of zones consisting of normally grown bacterial cells without revertant colonies was the criterion of antimutagenicity.

Among 303 kinds of different plant extracts, 18 showed

Table I. List of Plants for Assay

Tissues of the plant body used for extraction are indicated as follows.

At, aerial tuber; C, caulis; Fr, fruit; L, leaf; R, root; S, seed; St, stem; T, twig.; FL, flower.

1. L, F1: Aconitum chinense (Torikabuto)

2. L: Aquilegia flabellata (Odamaki)

3. L: Coptis japonica forma brachypetala (Seribaôren)

4. R: Coptis japonica forma brachypetala (Seribaôren)

5. L: Paeonia suffruticosa (Botan)

6. L: Ranunculus quelpaertensis (Kitunenobotan)

7. L: Ranunculus japonica (Umanoasigata)

8. L, St: Ranunculus sceleratus (Tagarasi)

9. L, St: Ranunculus nipponicus var. major (Baikamo)

10. F1: Akebia quinata (Akebi)

11. L: Stauntonia hexaphylla (Mube)

12. L: Mahonia japonica (Hiiraginanten)

13. L: Nandina domestica (Nanten)

14. Fr: Nandina domestica (Nanten)

15. L: Lindera glauca (Yamakôbasi)

16. T: Lindera glauca (Yamakôbasi)

17. L: Lindera obtusiloba (Dankôbai)

18. L, Fr: Lindera strychinfolia (Tendaiuyaku)

19. L: Cinnamomum sieboldii (Nikkei)

20. L: Cinnamomum camphora (Kusunoki)

21. L: Actinodaphne longifolia (Baribarinoki)

22. L: Parabenzoin trilobum (Siromozi)

23. L, F1: Papaver rhoeas (Hinagesi)
24. C: Macleaya cordata (Takenigusa)

25. L: Macleaya cordata (Takenigusa)

26. S: Chelidonium majus var. asiaticum (Kusanoo)

27. L: Chelidonium majus var. asiaticum (Kusanoo)

28. L, C: Eschscholzia californica (Hanabisiso)

29. F1, Fr: Corydalis platycarpa (Kikeman)

30. L: Corydalis platycarpa (Kikeman)

31. F1: Corydalis incisa (Murasakikikeman)

32. L: Corydalis incisa (Murasakikikeman)

33. Fr: Elaeocarpus photiniifolius (Simahorutonoki)

34. S: Gossypium indisum (Watanomi)

35. L: Malva sylvestris var. mauritiana (1) (Zeniaoi)

36. L: Malva sylvestris var. mauritiana (2) (Zeniaoi)

37. L, T: Hibiscus syriacus (Mukuge)

38. L: Cleyera japonica (Sakaki)

39. L: Camellia japonica (Tubaki)

40. F1: Camellia japonica (Tubaki)

41. L: Gracinia subelliptica (Fuguki)

42. L: Calophyllum inophyllum (Terihaboku)

43. F1: Hypericum patulum (1) (Kinsibai)

44. L: Hypericum patulum (1) (Kinsibai)

45. L: Hyperium patulum (2) (Kinsibai)

46. L: Eucalyptus globulus (Yûkari) 
TABle I (continued).

\begin{tabular}{|c|c|}
\hline 47. L, Young: Eucalyptus globulus (Yûkari) & 104. Fr: Liriope minor (Himeyaburan) \\
\hline 48. Fr: Eucalyptus globulus (Yûkari) & 105. L: Rohdea japonica (Omoto) \\
\hline 49. L.: Alangium plantanifolium var. (Urinoki) & 106. L: Xanthoxylum pipertum (Sansyo) \\
\hline 50. Fr: Punica granatum (Zakuro) & 107. Fr: Geranium nepalense (Gennosyôko) \\
\hline 51. L, C: Bixa orellana (Beninoki) & 108. L: Geranium nepalense (Gennosyôko) \\
\hline 52. L: Viola grypoceras (Tatitubosumire) & 109. L, C: Oxalis martiana (Murasakikatabami) \\
\hline 53. L: Psidium guajava (Banzirô) & 110. L, C, F1: Oxalis corniculata (Katabami) \\
\hline 54. Fr: Psidium guajava (Banzirô) & 111. Fr: Citrus tamurana (Konatumikan) \\
\hline 55. Fr: Aralia cordata (Udo) & 112. Fr: Citrus unshiu (Unsyûmikan) \\
\hline 56. L: Dendropanax trifidus (Kakuremino) & 113. L: Skimmia japonica (Miyamasikimi) \\
\hline 57. Fr: Fatsia japonica (Yatude) & 114. F1: Skimmia japonica (Miyamasikimi) \\
\hline 58. Fr: Fatsia japonica (Wakayama) (Yatude) & 115. Fr: Skimmia japonica (Miyamasikimi) \\
\hline 59. L: Fatsia japonica (Yatude) & 116. L: Phellodendron amurense (Kihada) \\
\hline 60. L: Cryptotaenia japonica (Mituba) & 117. T: Phellodendron amurense (Kihada) \\
\hline 61. L: Chamaele decumbens (Sentôsô) & 118. L, St: Maytenus diversifolia (Hariturumasaki) \\
\hline 62. L: Hydrocotyle sibthorpioides (Tidomegusa) & 119. L, C: Polygala japonica (Himehagi) \\
\hline 63. R, L: Panax Schinseng var. japonicas (Totibaninzin) & 120. L, T: Canarium album (Kanran) \\
\hline 64. L: Panax Schinseng (Tyosenninzin) & 121. L: Picrasma quassioides (Nigaki) \\
\hline 65. R: Daucus carota (Ninzin) & 122. Fr: Fortunella japonica (Kinkan) \\
\hline 66. L: Hedera rhombea (Kizuta) & 123. L, T: Toona sinensis (Tyantin) \\
\hline 67. Fr: Hedera rhombea (Kizuta) & 124. L: Rhododendron pentaphyllum (Akagitutuzi) \\
\hline 68. L, C: Cnidium officinale (Senkyû) & 125. L, C: Chrysosplenium grayanum (Yukinosita) \\
\hline 69. R: Cnidium officinale (Senkyû) & 126. L: Aleurites cordata (Aburagiri) \\
\hline 70. L, C: Foeniculum vulgare (Uikyô) & 127. L, C, F1: Mercurialis leiocarta (Yamaai) \\
\hline 71. L: Helwingia japonica (Hanaikada) & 128. L, T: Mallotus japonicus (Akamegasiwa) \\
\hline 72. L: Petroselinum crispum (Paseri) & 129. Fr: Ilex crenata (Inutuge) \\
\hline 73. L, F1: Briza maxima (Kobansô) & 130. L: Ilex crenata (Inutuge) \\
\hline 74. L, Fr: Hordeum vulgare var. hexastichon (Oomugi) & 131. L, T: Rhus orientalis (Tutaurusi) \\
\hline 75. L: Phyllostachys pubescens (Môsôtiku) & 132. L: Buxus microphylla var. suffruticosa (Tuge) \\
\hline 76. L, C, F1: Melica nutants (Komegaya) & 133. Fr: Buxus microphylla var. suffruticosa (Tuge) \\
\hline 77. L, C: Pinellia ternata (Karasubisyaku) & 134. L: Eucommia ulmoides (Totyû) \\
\hline 78. L: Żantedeschia aethiopica (Orandakaiu) & 135. L, T: Celastrus orbiculatus (Uruumemodoki) \\
\hline 79. R: Acorus gramineus (Sekisyô) & 136. S: Acer sp. (Kaede) \\
\hline 80. L: Acorus gramineus (Sekisyô) & 137. S: Litchi chinensis (Reisi) \\
\hline 81. L: Acorus calamus var. asiaticus (Syôbu) & 138. L: Litchi chinensis (Reisi) \\
\hline 82. Fr: Annas comosus (Painappuru) & 139. L: Litchi chinensis (Kanton) (Reisi) \\
\hline 83. L: Annas comosus (Painappuru) & 140. T: Litchi chinensis (Reisi) \\
\hline 84. L: Arisaema monophyllum (Hitotubanantenshô) & 141. L: Parthenocissus tricuspidata (Tuta) \\
\hline 85. F1: Arisaema monophyllum (Hitotubanantenshô) & 142. L, T: Vitis vinifera (Budô) \\
\hline 86. L: Rhaphidophora pinnata (Habukazura) & 143. L: Zizyphus jujuba (Sanebutonatume) \\
\hline 87. L: Tradescantia reflexa (Murasakituyukusa) & 144. L: Ajuga decumbens (1) (Kiransô) \\
\hline 88. C: Tradescantia reflexa (Murasakituyukusa) & 145. L: Ajuga decumbens (2) (Kiransô) \\
\hline 89. F1: Tradescantia reflexa (Murasakituyukusa) & 146. L: Ajuga decumbens (3) (Kiransô) \\
\hline 90. L: Allium tuberosum (Nira) & 147. R: Ajuga decumbens var. (Seiyo) (Seiyôkiranso) \\
\hline 91. L, C, F1: Tricyrtis hirta (Hototogisu) & 148. L: Vitex rotundifolia (Hamagô) \\
\hline 92. L, C: Asparagus cochinchinensis (Tenmondo) & 149. C: Vitex rotundifolia (Hamagô) \\
\hline 93. L: Mallotus japonicus (Akamegasiwa) & 150. L: Lantana camara (Rantana) \\
\hline 94. L: Lilium lancifolium (Oniuri) & 151. L: Callicarpa japonica (Murasakisikibu) \\
\hline 95. R: Lilium lancifolium (Oniyuri) & 152. L: Perilla frutescens (Siso) \\
\hline 96. At: Lilium lancifolium (Oniyuri) & 153. F1: Salvia officinalis (Sarubia) \\
\hline 97. L, C: Disporum sessile (Hôtyakusô) & 154. L, C: Lamium album var. barbatum (Odorikosô) \\
\hline 98. L: Disporum smilacinum (Tigoyuri) & 155. L: Glechoma hederacea (Kakidôsi) \\
\hline 99. R: Disporum smilacinum (Tigoyuri) & 156. L, C: Meehania urticifolia (Rasyômonkazura) \\
\hline 00. Fr: Asparagus officinalis (Asuparagasu) & 157. L: Rabdosia japonica (Hikiokosi) \\
\hline 01. R: Asparagus cochinchinensis (Tenmondô) & 158. L: Solanum lyratum (Hiyodorisyôgo) \\
\hline 02. L: Ophiopogon japonicus (1) (Ryûnohige) & 159. L: Hyoscyamus niger (Hiyosu) \\
\hline 03. L: Ophiopogon japonicus (2) (Zyanohige) & 160. R: Hyoscyamus niger (Hiyosu) \\
\hline
\end{tabular}


TABLE I (continued)

161. C: Scopolia japonica (Hasiridokoro)

162. L: Scopolia japonica (Hasiridokoro)

163. L: Lycium chinense (Kuko)

164. F1: Digitalis purpurea (Zikitarisu)

165. L, C: Veronica persica (Ooinunofuburi)

166. L, C: Mazus miquelii (Murasakisagigoke)

167. L, C: Vandellia crustacea (Urikusa)

168. F1: Paulownia tomentosa (Kiri)

169. L: Paulownia tomentosa (Kiri)

170. L, C: Plantago lanceolata (1) (Heraoobako)

171. R: Plantago lanceolata (2) (Heraoobako)

172. L, C: Plantago lanceolata (2) (Heraoobako)

173. L: Digitalis purpurea (Zikitarisu)

174. L: Gardenia jasminoides (Kutinasi)

175. L: Paederia scandens (Hekusokazura)

176. Fr: Paederia scandens (Hekusokazura)

177. Fr: Psychotria rubra (Ryûkyûaoki)

178. L: Psychotria rubra (Ryûkyûaoki)

179. L, C: Rubia codifolia L. var. Mungista (Akane)

180. L: Mitchella undulata (Turuaridôsi)

181. L: Galium spurium L. var echinospermon (Yaemugura)

182. L: Scaevola frutescens var. sericea (Kusatobera)

183. L, C: Sedum alboroseum (Benkeiso)

184. L: Sedum alboroseum (Benkeiso)

185. F1: Sedum subtile (Himerenge)

186. F1, C: Sedum japonicum (Menomannengusa)

187. L: Cardamine scutata (Oobatanetukebana)

188. C: Rorippa indica (Inugasira)

189. L: Mitella japonica (Tyarumerusô)

190. F1, C: Mitella japonica (Tyarumerasô)

191. L: Chrysosplenium japonicum (Yamanekonomesô)

192. L: Chrysosplenium grayanum (Nekonomesô)

193. L: Saxifraga stolonifera (Yukinosita)

194. L: Sedum bulbiferum (Komotimannengusa)

195. L: Schizophragma hydrangeoides (Iwagarami)

196. L: Deutzia gracilis (Himeutugi)

197. L: Philadelphus satsumi (Baikautugi)

198. F1: Philadelphus satsumi (Baikautugi)

199. Fr: Pittosporum tobira (Tobera)

200. L: Hydrangea macrophylla (Amatya)

201. L, C: Itea japonica (Zuina)

202. F1: Itea japonica (Zuina)

203. Fr: Pyracantha angustifolia (Pirakansasu)

204. L: Pyracantha angustifolia (Pirakansasu)

205. L: Spiraea cantoniensis (Kodemari)

206. L: Spiraea nipponica (Simotuke)

207. L: Distylium racemosum (Yusunoki)

208. Fr: Distylium racemosum (Yusunoki)

209. L: Rosaceae (Bara)

210. L: Rubus parvifolius (Nawasiroitigo)

211. L: Rubus hirsutus (Kusaitigo)

212. L: Crataegus cuneata (Sanzasi)

213. L: Albizia julibrissin (Nemunoki)

214. L, C: Trifolium patens (Himetumegusa)

215. L: Eriobotrya japonica (Biwa)

216. S: Eriobotyra japonica (Biwa)
217. L: Derris malaccensis (Tatitoba)

218. L: Trifolium repens (Sirotumegusa)

219. L: Acacia confusa (Sôsizyu)

220. T: Sophora sp. (Sidareenzyu)

221. L: Sophora sp. (Sidareenzyu)

222. L: Pueraria lobata (Kuzu)

223. L, C: Vicia satiba (Karasunoendô)

224. L, C: Vicia hirsuta (Suzumenoendô)

225. L, C: Astragalus sinicus (Renge)

226. L: Phaseolus sp. (Kuzuingen)

227. F1: Erythrina crista-galli (Amerikadeigo)

228. L: Leguminosa (1) (Mame-ka)

229. L: Leguminosa (2) (Mame-ka)

230. L: Leguminosa (3) (Mame-ka)

231. Fr: Pongamia pinnata (Kuroyona)

232. L: Lonicera japonica (Suikazura)

233. F1: Lonicera japonica (Suikazura)

234. F1: Viburnum sieboldi (Gomagi)

235. L: Viburnum sieboldi (Gomagi)

236. F1: Viburnum wrightii (Miyamagamazumi)

237. L: Viburnum wrightii (Miyamagamazumi)

238. L: Sambucus sieboldiana (Niwatoko)

239. L: Weigela hortensis (Takoutugi)

240. F1: Weigela coraeensis (Hakoneutugi)

241. L: Weigela coraeensis (Hakoneutugi)

242. L: Valeriana sambucifolia (Kanokosô)

243. L: Melothria japonica (Okinawa) (Okinawasuzumeuri)

244. Fr: Melothria japonica (Okinawa) (Okinawasuzumeuri)

245. Fr: Melothria japonica (Suzumeuri)

246. L: Gnaphalium affine (Hahakogusa)

247. L, C: Erigeron philadelphicus (Haruzyoon)

248. F1: Erigeron philadelphicus (Haruzyoon)

249. L: Erigeron thunbergii (Azumagiku)

250. F1: Erigeron thunbergii (Azumagiku)

251. L: Ligularia tussilaginea (Tuwabuki)

252. F1: Petasites japonicus (Fuki)

253. L: Petasites japonicus (Fuki)

254. L, C: Matricaria chamomilla (Kamiture)

255. L: Chrysanthemum cinerariaefolium (Sirobanamusyokegiku)

256. L: Achillea sibirica (Nokogiriso)

257. L: Qenothera tetraptera (Tukimisô)

258. L: Oenothera strueta (Matuyoigusa)

259. F1: Taraxacum officinale (Seiyôtanpopo)

260. L: Atractylis ovata (Okera)

261. L, C: Calendula officinalis (Kinsenka)

262. F1: Calendula officinalis (Kinsenka)

263. L: Artemisia vulgaris var. indica (Yomogi)

264. L: Houttuynia cordata (Dokudami)

265. L: Saururus chinensis (1) (Hangesyô)

266. R: Saururus chinensis (2) (Hangesyô)

267. L: Saururus chinensis (2) (Hangesyô)

268. L: Piper kadzura (Fûtôkazura)

269. L: Piper migrum (Kosyô)

270. L: Myrica rubra (Yamamomo) 
TABLE I (continued).

271. L, F1: Salix gracilistyla (Kawayanagi)

272. L: Chloranthus glaber (Senryô)

273. Fr: Chloranthus glaber (Senryô)

274. L: Ficus carica (Itiziku)

275. L: Quercus phillyraeoides (Ubamegasi)

276. Fr: Alnus sieboldiana (Oobayasyabusi)

277. L: Alnus sieboldiana (Oobayasyabusi)

278. L: Polygonum cuspidatum (Itadori)

279. Fr: Polygonum filiforme (Mizuhiki)

280. L, C: Persicaria longiseta (Inutade)

281. L: Nanocnid japonica (Katensô)

282. Fr: Humulus scandens (Kanamugura)

283. L: Humulus scandens (Kanamugura)

284. L: Sagina japonica (Tumekusa)

285. L: Silene gallica (Sirobanamantema)

286. L: Phytolacca americana (Amerikayamagobô)

287. C: Phytolacca americana (Amerikayamagobo)
288. L: Achyranthes japonica (Inokozuti)

289. Fr: Kadsura japonica (Sanekazura)

290. Fr: Schizandra nigra (Matubusa)

291. F1: Ficus retusa (Gazyumaru)

292. S: Ficus retusa (Gazyumaru)

293. L: Ficus retusa (Gazyumaru)

294. L: Metasequoia glyptostroboides (Metasekoia)

295. L: Tripetaleia paniculata (Hotutuzi)

296. Fr: Aucuba japonica (Aoki)

297. L: Lygodium japonicum (Kanikusa)

298. L: Fagara ailanthoides (Karasunosansyô)

299. L: Ginkgo biloba (Ityô)

300. L: Lysimachia japonicus (Konasubi)

301. S: Euphoria longana (Ryûgan)

302. L: Anemone coronaria (Anemone)

303. L: Anemone nikoensis (Itirinsô)
Table II. List of Positive Samples That Showed Antimutagenicities

\begin{tabular}{|c|c|c|}
\hline \multirow[b]{2}{*}{$\begin{array}{c}\text { The sample } \\
\text { numbers }\end{array}$} & \multicolumn{2}{|c|}{ Assay systems } \\
\hline & $\begin{array}{c}\text { E. coli } \\
\text { WP2 trp }\end{array}$ & $\begin{array}{c}\text { B. subtilis } \\
\text { NIG1125 } \\
\text { (his met) }\end{array}$ \\
\hline 5 & + & \\
\hline 6 & + & \\
\hline 7 & ++ & \\
\hline 8 & +++ & \\
\hline 17 & + & \\
\hline 18 & + & \\
\hline 25 & & ++ \\
\hline 53 & + & \\
\hline 63 & ++ & + \\
\hline 107 & & ++ \\
\hline 108 & & ++ \\
\hline 121 & & ++ \\
\hline 124 & + & ++ \\
\hline 126 & ++ & ++ \\
\hline 131 & & + \\
\hline 164 & + & \\
\hline 167 & & + \\
\hline 180 & & + \\
\hline 189 & ++ & + \\
\hline 207 & ++ & +++ \\
\hline 208 & ++ & +++ \\
\hline 276 & + & +++ \\
\hline 294 & + & + \\
\hline 302 & ++ & \\
\hline 303 & ++ & \\
\hline
\end{tabular}

+: Detection of antimutagenicity zones whose length are $0 \sim 5 \mathrm{~mm}$ by at least two experiments.

$++:$ Ibid., $5 \sim 7 \mathrm{~mm}$.

+++ : Ibid., more than $7 \mathrm{~mm}$. positive responses to $E$. coli $\mathrm{B} / \mathrm{r} \mathrm{WP} 2$ and 16 to $B$. subtilis 1125 (Table II). Among these positive samples, only 8 responded simultaneously to both strains.

These results indicate that the antimutagenic factors are only detectable in limited species of plants. It is also suggested that no universal bio-antimutagenic ingredient exists in the plant kingdom as measured by the present assay systems. Since no common factor could reduce induced and spontaneous mutabilities of the strains of $E$. coli $\mathrm{B} / \mathrm{r}$ WP2 and $B$. subtilis 1125 , it is difficult to suppose that a certain DNA-polymerase III is involved universally in the UV-induced SOS function. It is noted that the antimutagenicities of certain plant ingredients possessing antioxidant capabilities show quite different spectra from those shown in the present data (M. Namiki and $T$. Osawa, personal communication).

Chemical isolation studies are under way to elucidate further mechanisms of antimutagenesis involved.

Acknowledgment. We thank Mr. Hirotaka Kato and his group very much for their collaboration in the screening studies. The present research was srpported in part by Grants-in-Aid from the Ministry of Education, Science and Culture and the Ministry of Welfare of Japan.

\section{REFERENCES}

1) T. Kada, T. Inoue and M. Namiki, "Environmental Mutagenesis, Carcinogenesis, and Plant Biology," Vol. I, ed. E. J. Klekowski, Jr., Praeger, New York, 1982, p. 135.

2) H. Minakata, H. Komura, K. Nakanishi and T. Kada, Mutation Res., 116, 317 (1983).

3) K. Kakinuma, J. Koike, K. Kotani, N. Ikekawa, T. Kada and M. Nomoto, Agric. Biol. Chem., 48, 1905 (1984). 
4) K. Kakinuma, Y. Okada, N. Ikekawa, T. Kada and M. Nomoto, Agric. Biol. Chem., 48, 1647 (1984).

5) T. Kada and N. Kanematsu, Proc. Japan Acad., 54B, 234 (1978).
6) T. Inoue, Y. Ohta, Y. Sadaie and T. Kada, Mutation Res., 91, 41 (1981).

7) B. A. Bridges and R. P. Mottershead, Mol. Gen. Genet., 162, 35 (1978). 